\title{
SIMULTANEOUS OCCURRENCE OF SLIDING AND CROSSING LIMIT CYCLES IN PIECEWISE LINEAR PLANAR VECTOR FIELDS
}

\author{
JOAO L. CARDOSO ${ }^{1}$, JAUME LLIBRE ${ }^{2}$, DOUGLAS D. NOVAES ${ }^{3}$ AND DURVAL J. TONON ${ }^{1}$
}

\begin{abstract}
In the present study we consider planar piecewise linear vector fields with two zones separated by the straight line $x=0$. Our goal is to study the existence of simultaneous crossing and sliding limit cycles for such class of vector fields. First, we provide a canonical form for these systems assuming that each linear system has center, a real one for $y<0$ and a virtual one for $y>0$, and such that the real center is a global center. Then working with a first order piecewise linear perturbation we obtain piecewise linear differential systems with three crossing limit cycles. Second, we see that a sliding cycle can be detected after a second order piecewise linear perturbation. Then imposing the existence of a sliding limit cycle we prove that only one more crossing limit cycle can appear. Finally, we also characterize the stability of the higher amplitude limit cycle and of the infinity. The main techniques used in our proofs are the Melnikov functions, the Extended Chebyshev systems and the Bendixson transformation.
\end{abstract}

\section{IntRoduCtion AND STATEMENT OF THE MAIN RESUlts}

For a given differential system a limit cycle is a periodic orbit isolated in the set of all periodic orbits of the system. One of the main problems of the qualitative theory of planar differential systems is determining the existence of limit cycles. A center is a singular point $p$ that possesses a neighborhood $U$ such that $U \backslash\{p\}$ is filled by periodic solutions. A classical way to produce and study limit cycles is by perturbing the periodic solutions of a center. This problem has been studied intensively for continuous planar differential systems, see for instance, [5] and the references therein.

The main objective of this paper is to study the limit cycles that can bifurcate from a center of a discontinuous piecewise linear differential systems with two zones separated by the straight line $x=0$ when the center is perturbed inside the class of all discontinuous piecewise linear differential systems with two zones separated by $x=0$.

The study of the piecewise linear differential systems goes back to Andronov and coauthors [1]. In the present days these systems continue receiving a considerable attention, mainly due to their applications. Indeed, such systems are widely used to model many real processes and different modern devices, see for instance the book [3] and the references therein.

The case of continuous piecewise linear systems with two regions separated by a straight line is the simplest possible configuration of piecewise linear systems. We note that even in this simple case, establishing the existence of at most one limit cycles was a difficult task, see [6], and [12] for a shorter proof. There are two reason for that misleading simplicity of

2010 Mathematics Subject Classification. 34C05, 34C07, 37G15.

Key words and phrases. non-smooth differential system, limit cycle, piecewise linear differential system. 
piecewise linear systems: First, whereas it is easy to compute the solutions in any linear region, the time that each orbit requires to pass from one linear region to the other is usually not computable. Second, the increasing number of parameters.

Discontinuous piecewise linear systems with two regions separated by a straight line have received a lot of attention during these last years, see for instance $[9,10,13,14,16,17]$ among other papers. In [9], the authors conjectured that piecewise linear systems with two regions separated by a straight line could have at most two limit cycles. Later on in [10], the authors provided numerical evidences on the existence of three limit cycles, which was analytically proved in [16].

In the present study, we consider planar piecewise linear vector fields with two zones separated by the straight line $x=0$. Firstly, we provide a canonical form for these systems assuming that each linear system has center, a real one for $y<0$ and a virtual one for $y>0$, such that the real center is a global center. Then, proceeding with a first order piecewise linear perturbation, we study the bifurcation of crossing limit cycles. In short, it is shown that any configuration of 1, 2 or 3 limit cycles can bifurcate from the periodic solutions of the unperturbed system. We also study the stability of the higher amplitude crossing limit cycle and the infinity. Secondly, we see that a sliding cycle can be detected after a second order piecewise linear perturbation. Finally, imposing the existence of a sliding limit cycle we prove that only one more crossing limit cycle appears.

1.1. Setting the problem. Consider the following planar piecewise linear differential systems with two zones separated by the straight line $\Sigma=\{(x, y) ; x=0\}$

$$
\left(\begin{array}{c}
\dot{x} \\
\dot{y}
\end{array}\right)=W_{\varepsilon}(x, y)=\left\{\begin{array}{l}
M_{\varepsilon}^{+}\left(\begin{array}{c}
x \\
y
\end{array}\right)+\left(\begin{array}{c}
u_{1, \varepsilon}^{+} \\
u_{2, \varepsilon}^{+}
\end{array}\right) \quad \text { if } \quad x \geq 0, \\
M_{\varepsilon}^{-}\left(\begin{array}{l}
x \\
y
\end{array}\right)+\left(\begin{array}{c}
u_{1, \varepsilon}^{-} \\
u_{2, \varepsilon}^{-}
\end{array}\right) \quad \text { if } \quad x \leq 0,
\end{array}\right.
$$

where $M_{\varepsilon}^{ \pm}$is one-parameter family of $2 \times 2$ real matrices and $u_{1, \varepsilon}^{ \pm}, u_{2, \varepsilon}^{ \pm} \in \mathbb{R}$. Here, the dot denotes derivative with respect to the time variable $t$. We define $\Sigma^{+}=\{(x, y) ; x>0\}$ and $\Sigma^{-}=\{(x, y) ; x<0\}$ and denote the piecewise linear vector field associated to system (1) by $W_{\varepsilon}=\left(W_{\varepsilon}^{+}, W_{\varepsilon}^{-}\right)$, where $W_{\varepsilon}^{+}$and $W_{\varepsilon}^{-}$are defined in $\Sigma^{+}$and $\Sigma^{-}$, respectively.

Let $p^{ \pm}$be the singular points of $W_{0}^{ \pm}$. We say that a singular point $p^{ \pm}$of $W_{0}^{ \pm}$is real (respectively virtual) if $p^{+} \in\{(x, y) ; x \geq 0\}$ or $p^{-} \in\{(x, y) ; x \leq 0\}$ (respectively if $p^{+} \in$ $\{(x, y) ; x \leq 0\}$ or $\left.p^{-} \in\{(x, y) ; x \geq 0\}\right)$. Assume that system (1) satisfies

$\left(H_{1}\right) p^{-}$is a center for the system $W_{0}^{-}$and $p^{-} \in \Sigma^{-}$, i.e, $p^{-}$is a real singular point.

$\left(H_{2}\right) p^{+}$is a center for the system $W_{0}^{+}$and $p^{+} \in \Sigma^{-}$, i.e, $p^{+}$is a virtual singular point.

$\left(H_{3}\right) p^{-}$is global center for the system $W_{0}$, see Figure 1.

1.2. Canonical Form. The next result provides a canonical form for the piecewise linear vector fields (1) having a center and it is proved in Section 3. Fixing $\varepsilon=0$, we denote

$$
M_{0}^{ \pm}=\left(\begin{array}{cc}
m_{11}^{ \pm} & m_{12}^{ \pm} \\
m_{21}^{ \pm} & -m_{11}^{ \pm}
\end{array}\right)
$$




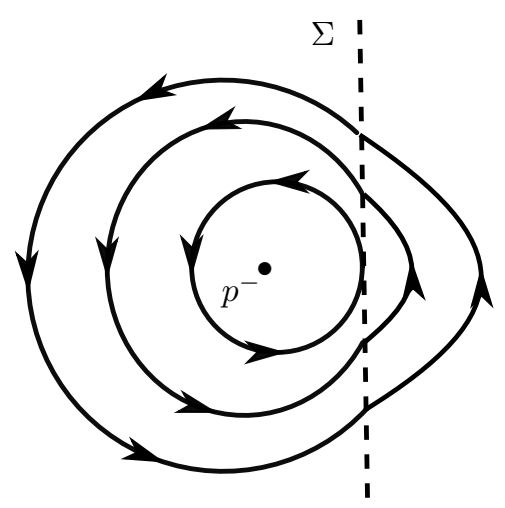

Figure 1. The global center for the system $W_{0}$.

Proposition 1. Consider the piecewise linear vector field $W_{\varepsilon}(x, y)$ given in (1) and assume that $W_{\varepsilon}(x, y)$ satisfies hypotheses $\left(H_{1}\right),\left(H_{2}\right)$ and $\left(H_{3}\right)$. Then, there exists a change of coordinates $(t, x, y) \mapsto(\tilde{t}, x, \tilde{y})$ where the piecewise linear vector field (1) can be written as $\left(x^{\prime}, \tilde{y}^{\prime}\right)^{T}=Z_{\varepsilon}(x, \tilde{y})$, where

$$
Z_{0}(x, \tilde{y})=\left\{\begin{array}{l}
Z_{0}^{+}(x, \tilde{y})=A^{+}\left(\begin{array}{l}
x \\
\tilde{y}
\end{array}\right)+\left(\begin{array}{l}
0 \\
d
\end{array}\right) \quad \text { if } \quad x \geq 0, \\
Z_{0}^{-}(x, \tilde{y})=A^{-}\left(\begin{array}{l}
x \\
\tilde{y}
\end{array}\right)+\left(\begin{array}{l}
0 \\
e
\end{array}\right) \quad \text { if } \quad x \leq 0,
\end{array}\right.
$$

$A^{+}=\left(\begin{array}{cc}a & b \\ c & -a\end{array}\right), A^{-}=\left(\begin{array}{cc}0 & -1 \\ 1 & 0\end{array}\right)$ with $\rho=\sqrt{\left|\left(m_{11}^{-}\right)^{2}+m_{12}^{-} m_{21}^{-}\right|}, a=\frac{1}{\rho}\left(m_{11}^{+}\right.$ $\left.-\frac{m_{11}^{-} m_{12}^{+}}{m_{12}^{-}}\right), b=-\frac{m_{12}^{+}}{m_{12}^{-}}, c=\frac{1}{\rho^{2}}\left(\frac{\left(m_{11}^{-}\right)^{2} m_{12}^{+}}{m_{12}^{-}}-2 m_{11}^{-} m_{11}^{+}-m_{12}^{+} m_{21}^{+}\right), d=-\frac{m_{12}^{+} u_{2}^{+}}{\rho}$ and $e=-\frac{m_{12}^{-} u_{2}^{-}}{\rho}$. Furthermore, the parameters of this canonical form satisfy

$$
b<0, c>0, d>0, e>0, a^{2}+b c<0 .
$$

In general we can write

$$
Z_{\varepsilon}(x, y)=Z_{0}(x, y)+\varepsilon Z_{1}(x, y)+\varepsilon^{2} Z_{2}(x, y)+\mathcal{O}\left(\varepsilon^{3}\right)
$$

where

$$
Z_{1}(x, y)=\left\{\begin{array}{c}
B^{+}\left(\begin{array}{c}
x \\
y
\end{array}\right)+v^{+} \text {if } \quad x \geq 0, \\
B^{-}\left(\begin{array}{c}
x \\
y
\end{array}\right)+v^{-} \text {if } \quad x \leq 0,
\end{array}\right.
$$


and

$$
Z_{2}(x, y)=\left\{\begin{array}{c}
C^{+}\left(\begin{array}{l}
x \\
y
\end{array}\right)+w^{+} \quad \text { if } \quad x \geq 0, \\
C^{-}\left(\begin{array}{l}
x \\
y
\end{array}\right)+w^{-} \text {if } \quad x \leq 0,
\end{array}\right.
$$

with $B^{ \pm}=\left(\begin{array}{cc}b_{11}^{ \pm} & b_{12}^{ \pm} \\ b_{21}^{ \pm} & b_{22}^{ \pm}\end{array}\right), \quad v^{ \pm}=\left(\begin{array}{c}v_{1}^{ \pm} \\ v_{2}^{ \pm}\end{array}\right), \quad C^{ \pm}=\left(\begin{array}{cc}c_{11}^{ \pm} & c_{12}^{ \pm} \\ c_{21}^{ \pm} & c_{22}^{ \pm}\end{array}\right), \quad w^{ \pm}=\left(\begin{array}{c}w_{1}^{ \pm} \\ w_{2}^{ \pm}\end{array}\right)$. We denote the piecewise smooth vector field $Z_{\varepsilon}$ by $Z_{\varepsilon}=\left(Z_{\varepsilon}^{+}, Z_{\varepsilon}^{-}\right)$, where $Z_{\varepsilon}^{ \pm}$is defined in $\Sigma^{ \pm}$.

1.3. Crossing limit cycles. For the piecewise linear differential system (2) we obtain an upper bound for the number of crossing limit cycles bifurcating from the periodic solutions of the unperturbed system. We define

$$
\begin{aligned}
& K_{0}=\frac{2 d\left(\xi^{2}\left(2 v_{1}^{-} b-b e\left(b_{11}^{-}+b_{22}^{-}\right)+2 v_{1}^{+}\right)+b d\left(b_{11}^{+}+b_{22}^{+}\right)\right)}{e \xi}, \\
& K_{1}=b e \xi^{2}\left(b_{11}^{-}+b_{22}^{-}\right) \\
& K_{2}=-\frac{b d^{2}\left(b_{11}^{+}+b_{22}^{+}\right)}{e \xi}
\end{aligned}
$$

where $a^{2}+b c=-\xi^{2}$, with $\xi>0$.

Theorem A. Suppose $Z_{\varepsilon}(x, y)$ is a piecewise linear vector field with two zones separated by the straight line $x=0$ such that hypotheses $\left(H_{1}\right),\left(H_{2}\right)$ and $\left(H_{3}\right)$ are satisfied by $Z_{0}$. Without loss of generality, assume that $Z_{\varepsilon}$ writes as (2). Then, the following statements hold.

(a) There exist elections of the parameters $K_{0}, K_{1}$, and $K_{2}$ for which system $Z_{\varepsilon}$ has any configuration of 1,2 , or 3 crossing limit cycles taking into account their multiplicity.

(b) The highest amplitude limit cycle (when it exists) and the infinity have opposite stability. More precisely, the highest amplitude limit cycle is stable (resp. unstable) and the infinity is an unstable (resp. stable) periodic orbit provided that $\xi\left(b_{11}^{-}+\right.$ $\left.b_{22}^{-}\right)+b_{11}^{+}+b_{22}^{+}<0$ (resp. $\left.\xi\left(b_{11}^{-}+b_{22}^{-}\right)+b_{11}^{+}+b_{22}^{+}>0\right)$.

(c) The lowest amplitude limit cycle (when it exists) is stable (resp. unstable) provided that $b_{11}^{-}+b_{22}^{-}<0$, or $b_{11}^{-}+b_{22}^{-}=0$ and $b v_{1}^{-}+v_{1}^{+}>0$ (resp. $b_{11}^{-}+b_{22}^{-}>0$, or $b_{11}^{-}+b_{22}^{-}=0$ and $\left.b v_{1}^{-}+v_{1}^{+}<0\right)$.

Theorem A is proved in Subsection 3.3.

We note that it is well known that piecewise linear differential system with two regions separated by a straight line can exhibit 3 crossing limit cycles. The first numerical evidence of that was given in [10] by Huan and Yang. Then, in [16], Llibre and Ponce established an analytical proof of the existence of 3 limit cycles. After that, many authors have provided different examples of those systems having 3 limit cycles. For instance, in [4] Buzzi et al., using a seventh order piecewise linear perturbation, proved that 3 limit cycles bifurcate from a linear center. In [13], Llibre et al. proved that 3 limit cycles bifurcates from a 
piecewise linear center, but using only a first order piecewise linear perturbation. In the present paper, Theorem A also guarantees the bifurcation of 3 crossing limit cycles after a first order perturbation. In the next subsection we shall see that a second order perturbation allows the study of sliding limit cycles.

1.4. Sliding and escaping limit cycles. We recall that a sliding/escaping limit cycle is a closed orbit composed by segments of orbits of the sliding vector field $Z^{s}$ (see Subsection 2.1) and $Z^{+}$and/or $Z^{-}$. We must mention that this kind of limit cycle has been considered before, see for instance [7].

Consider a piecewise linear vector field $Z$ and assume that the sliding/escaping region (see Subsection 2.1) is bounded. A sliding/escaping limit cycle of $Z$ is a closed trajectory composed by trajectories of the sliding vector field $Z^{s}, Z^{+}$, and/or $Z^{-}$. More precisely, we say that a sliding/escaping limit cycle is: of Type $I$ if it is composed by trajectories of $Z^{s}$, and $Z_{\varepsilon}^{+}$or $Z_{\varepsilon}^{-}$; and of Type $I I$ if it is composed by trajectories of $Z^{s}, Z_{\varepsilon}^{+}$and $Z_{\varepsilon}^{-}$, see Figure 2 .

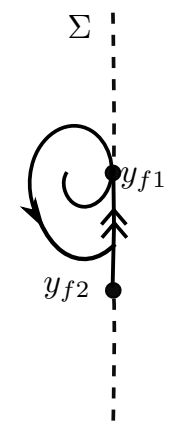

Type I

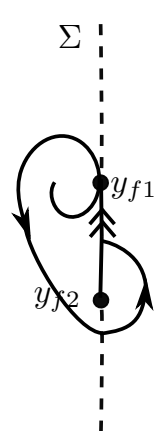

Type II

Figure 2. Types of sliding limit cycles.

Since we are dealing with piecewise linear vector fields, then the number of fold points for each vector field $Z_{\varepsilon}^{+}$and $Z_{\varepsilon}^{-}$is at most one. Therefore, the system can have at most one sliding/escaping limit cycle.

Theorem B. Suppose $Z_{\varepsilon}(x, y)$ is a piecewise linear vector field with two zones separated by the straight line $x=0$ such that hypotheses $\left(H_{1}\right),\left(H_{2}\right)$ and $\left(H_{3}\right)$ are satisfied by $Z_{0}$. Without loss of generality, assume that $Z_{\varepsilon}$ writes as (2).

(1) Additionally, suppose that $b_{11}^{-}=-b_{22}^{-}, b v_{1}^{-}+v_{1}^{+}<0$ and $0<a<d+b e$. Then, the following statements hold.

(a) If $0<c_{11}^{-}+c_{22}^{-}<\frac{\left(v_{1}^{-} b+v_{1}^{+}\right)^{2}}{2 b^{2} e^{2} \pi}$ then system $Z_{\varepsilon}$ admits a sliding cycle of Type I.

(b) If $\frac{\left(v_{1}^{-} b+v_{1}^{+}\right)^{2}}{2 b^{2} e^{2} \pi}<c_{11}^{-}+c_{22}^{-}<\frac{2\left(v_{1}^{-} b+v_{1}^{+}\right)^{2}}{b^{2} e^{2} \pi}$ then system $Z_{\varepsilon}$ admits a sliding cycle of Type $I I$.

(2) Conversely, suppose that $b_{11}^{-}=-b_{22}^{-}, b v_{1}^{-}+v_{1}^{+}>0, a<0$ and $0<d+b e$. Then, the following statements hold. 
(a) If $-\frac{\left(v_{1}^{-} b+v_{1}^{+}\right)^{2}}{2 b^{2} e^{2} \pi}<c_{11}^{-}+c_{22}^{-}<0$ then system $Z_{\varepsilon}$ admits a escaping cycle of Type $I$.

(b) If $c_{11}^{-}+c_{22}^{-}<-\frac{\left(v_{1}^{-} b+v_{1}^{+}\right)^{2}}{2 b^{2} e^{2} \pi}$ then system $Z_{\varepsilon}$ admits a escaping cycle of Type II.

Theorem B is proved in Subsection 3.4

1.5. Simultaneity. The next result provides conditions on the parameters of the vector field $Z_{\varepsilon}$ for the simultaneous occurrence of crossing and sliding/escaping limit cycles.

Theorem C. Under the assumptions of Theorem $B$ the following statements hold.

(1.a) If $0<c_{11}^{-}+c_{22}^{-}<\frac{\left(v_{1}^{-} b+v_{1}^{+}\right)^{2}}{2 \pi b^{2} e^{2}}$ then $Z_{\varepsilon}$ possesses a sliding limit cycle of type $I$ and at most one more crossing limit cycle.

(1.b) If $\frac{\left(v_{1}^{-} b+v_{1}^{+}\right)^{2}}{2 b^{2} e^{2} \pi}<c_{11}^{-}+c_{22}^{-}<\frac{2\left(v_{1}^{-} b+v_{1}^{+}\right)^{2}}{b^{2} e^{2} \pi}$ then $Z_{\varepsilon}$ possesses a sliding limit cycle of type II and at most one more crossing limit cycle.

(2.a) If $-\frac{\left(v_{1}^{-} b+v_{1}^{+}\right)^{2}}{2 b^{2} e^{2} \pi}<c_{11}^{-}+c_{22}^{-}<0$ then $Z_{\varepsilon}$ possesses a escaping limit cycle of type $I$ and at most one more crossing limit cycle.

(2.b) If $c_{11}^{-}+c_{22}^{-}<-\frac{\left(v_{1}^{-} b+v_{1}^{+}\right)^{2}}{2 b^{2} e^{2} \pi}$ then $Z_{\varepsilon}$ possesses a escaping limit cycle of type II and at most one more crossing limit cycle.

Moreover, there exist parameters of $Z_{\varepsilon}$ for which the above crossing limit cycle exists, see Figure 3.

Theorem C is proved in Subsection 3.5.
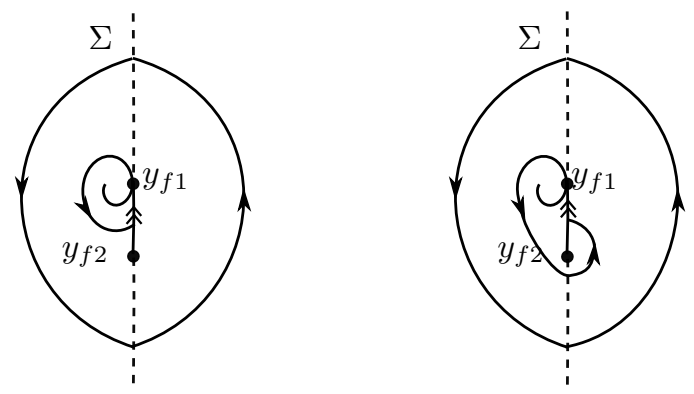

FiguRE 3. Simultaneous occurrence of crossing and sliding limit cycles for a 2-order linear perturbation of a piecewise linear center.

This paper is organized as follows. In Section 2 we provide some preliminary results. Section 3 is devoted to the proofs of our main results. Finally, in Section 4 we present some examples of piecewise linear vector fields for which the upper bounds of crossing and sliding/escaping limit cycles are reached. 


\section{Preliminaries}

This section is devoted to present some basic notions on nonsmooth vector fields and all the tools needed to prove our main results. Firstly, we introduce the concept of Filippov system. Then, some results on extended Chebyshev systems are presented. This results are important to bound the number of limit cycles. Finally, we discuss the Bendixon transformation which allows the study of the stability of the infinity.

2.1. Filippov's convention. Let $h: \mathbb{R}^{2} \rightarrow \mathbb{R}$ a differentiable function for which 0 is a regular value. Consider the piecewise smooth vector field

$$
Z(x, y)= \begin{cases}Z^{+}(x, y), & h(x, y)>0 \\ Z^{-}(x, y), & h(x, y)<0\end{cases}
$$

The switching manifold is given by $\Sigma=h^{-1}(0)$. Notice that $h(x, y)=x$ for system (1). Consider the Lie's derivative $Z^{ \pm} h(p)=\left\langle Z^{ \pm}, \nabla h\right\rangle$, where $\langle\cdot, \cdot\rangle$ is the canonical inner product in $\mathbb{R}^{2}$. Then, according to Filippov's conventions (see [20]), the following regions on $\Sigma$ are distinguished: Crossing Region: $\Sigma^{c}=\left\{p \in \Sigma ;\left(Z^{+} h\right)(p) .\left(Z^{-} h\right)(p)>0\right\}$.; Sliding Region: $\Sigma^{s}=\left\{p \in \Sigma ;\left(Z^{+} h\right)(p)<0,\left(Z^{-} h\right)(p)>0\right\}$; and Escaping Region: $\Sigma^{e}=\{p \in$ $\left.\Sigma ;\left(Z^{+} h\right)(p)>0,\left(Z^{-} h\right)(p)<0\right\}$. The local trajectories of the points in $\Sigma^{s} \cup \Sigma^{e}$ follow the so called sliding vector field

$$
Z^{s}=\frac{Z^{-} h \cdot Z^{+}-Z^{+} h \cdot Z^{-}}{Z^{-} h-Z^{+} h}
$$

Then, the flow of $Z$ is obtained by the concatenation of flows of $Z^{+}, Z^{-}$, and $Z^{s}$.

We say that $p \in \Sigma$ is a fold point of $Z^{ \pm}$when $Z^{ \pm} h(p)=0$ and $\left(Z^{ \pm}\right)^{2} h(p)=Z^{ \pm} \cdot\left(Z^{ \pm} h\right)(p) \neq$ 0 . Moreover, $p$ is called a visible (resp. invisible) fold point of $Z^{ \pm}$if $Z^{ \pm} h(p)=0$ and $\left(Z^{ \pm}\right)^{2} h(p) \gtrless 0\left(\operatorname{resp} .\left(Z^{ \pm}\right)^{2} h(p) \lessgtr 0\right)$.

Remark 1. Assuming that $p_{0} \in \Sigma^{c}$ is an invisible fold point for both vector fields $Z^{+}$and $Z^{-}$, a first return map is well defined in a neighborhood of $p_{0}$. Indeed, from the Implicit Function Theorem, there exists a neighborhood $U$ of $p_{0}$ such that for each $p \in U \cap \Sigma$, there exists a smallest positive time $t(p)>0$ such that the trajectory $t \mapsto \phi_{Z^{+}}(t, p)$ of $Z^{+}$ through $p$ intercepts $\Sigma$ at a point $\widetilde{p}=\phi_{Z^{+}}(t(p), p)$. Then, define the positive half-return map associated to $Z^{+}$by $\gamma_{Z^{+}}:(\mathbb{R}, 0) \rightarrow(\mathbb{R}, 0)$ where $\gamma_{Z^{+}}(p)=\widetilde{p}$. Analogously, we define the positive half-return map associated to $Z^{-}$by $\gamma_{Z^{-}}:(\mathbb{R}, 0) \rightarrow(\mathbb{R}, 0)$. Thus, the first return map $\varphi_{Z}:(\Sigma, 0) \rightarrow(\Sigma, 0)$ is defined by the composition $\varphi_{Z}=\gamma_{Z^{-}} \circ \gamma_{Z^{+}}$.

2.2. Extended Chebyshev systems. An ordered set of complex-valued functions $\mathcal{F}=$ $\left(g_{0}, g_{1}, \ldots, g_{k}\right)$ defined on a proper real interval $I$ is an Extended Chebyshev system or ETsystem on $I$ if and only if any nontrivial linear combination of functions in $\mathcal{F}$ has at most $k$ zeros counting multiplicities. The set $\mathcal{F}$ is an Extended Complete Chebyshev system or an ECT-system on $I$ if and only if for any $s \in\{0,1, \ldots, k\}$ we have that $\left(g_{0}, g_{1}, \ldots, g_{s}\right)$ is an ET-system. For more details see the book of Karlin and Studden [11].

In order to prove that $\mathcal{F}$ is an ECT-system on $I$ it is necessary and sufficient to show that $W\left(g_{0}, g_{1}, \ldots, g_{s}\right)(t) \neq 0$ on $I$ for $0 \leq s \leq k$, where $W\left(g_{0}, g_{1}, \ldots, g_{s}\right)(t)$ denotes the 
Wronskian of the functions $\left(g_{0}, g_{1}, \ldots, g_{s}\right)$ with respect to the variable $t$. That is,

$$
W\left(g_{0}, \ldots, g_{s}\right)(t)=\operatorname{det}\left(\begin{array}{ccc}
g_{0}(t) & \cdots & g_{s}(t) \\
g_{0}^{\prime}(t) & \cdots & g_{s}^{\prime}(t) \\
\vdots & \ddots & \vdots \\
g_{0}^{(s)}(t) & \cdots & g_{s}^{(s)}(t)
\end{array}\right)
$$

In [21], the authors proved the following results:

Theorem $2([21])$. Let $\mathcal{F}=\left[g_{0}, g_{1}, \ldots, g_{n}\right]$ be an ordered set of $C^{\infty}$ functions $g_{j}:[a, b] \rightarrow \mathbb{R}$ for $j=0,1, \ldots, n$ such that there exists $\xi \in(a, b)$ with $W\left(g_{0}, g_{1}, \ldots, g_{n-1}\right)(\xi)=W_{n-1}(\xi) \neq$ 0. Then, the following statements hold.

(a) If $W_{n}(\xi) \neq 0$, then for each configuration of $m \leq n$ zeros, taking into account their multiplicity, there exists $f \in \operatorname{Span}(\mathcal{F})$ with this configuration of zeros.

(b) If $W_{n}(\xi)=0$ and $W_{n}^{\prime}(\xi) \neq 0$, then for each configuration of $m \leq n+1$ zeros, taking into account their multiplicity, there exists $f \in \operatorname{Span}(\mathcal{F})$ with this configuration of zeros.

Corollary 3 ([21]). Let $\mathcal{F}=\left[g_{0}, g_{1}, \ldots, g_{n}\right]$ be an ordered set of $C^{\infty}$ functions $g_{j}:[a, b] \rightarrow \mathbb{R}$ for $j=0,1, \ldots, n$. Assume that all the Wronskians are nonvanishing except $W_{n}(x)$, which has exactly one zero on $(a, b)$ and this zero is simple. Then, $Z(\mathcal{F})=n+1$ and for any configuration of $m \leq n+1$ zeros there exists an element in $\operatorname{Span}(\mathcal{F})$ realizing it.

2.3. The Bendixson transformation. The Bendixson transformation is a useful tool to analyze the stability of the infinity of planar vector fields. In what follows, following $[8,15]$, we shall discuss this transformation. Consider the differential systems

$$
\dot{x}=f(x, y, \varepsilon), \quad \dot{y}=g(x, y, \varepsilon),
$$

where $f, g$ are Lipschitz functions in the variables $(x, y)$ and $\varepsilon>0$ is a small parameter. Applying to system (4) the Bendixson transformation defined as

$$
\left(\begin{array}{l}
u \\
v
\end{array}\right)=\frac{1}{x^{2}+y^{2}}\left(\begin{array}{l}
x \\
y
\end{array}\right),
$$

we obtain an equivalent system whose local phase portrait at the origin is equivalent to the local phase portrait of system (4) in a neighborhood of the infinity.

Composing the Bendixson change of variables (5) with the polar coordinates $u=r \cos \theta$, $v=r \sin \theta$, we get the polar Bendixson transformation $x=(\cos \theta) / r, y=(\sin \theta) / r$. Applying this last transformation, system (4) becomes

$$
\begin{aligned}
& \dot{r}=R(r, \theta, \varepsilon)=-r^{2}\left[f\left(\frac{\cos \theta}{r}, \frac{\sin \theta}{r}, \varepsilon\right) \cos \theta+g\left(\frac{\cos \theta}{r}, \frac{\sin \theta}{r}, \varepsilon\right) \sin \theta\right], \\
& \dot{\theta}=\xi(r, \theta, \varepsilon)=-r\left[f\left(\frac{\cos \theta}{r}, \frac{\sin \theta}{r}, \varepsilon\right) \cos \theta-g\left(\frac{\cos \theta}{r}, \frac{\sin \theta}{r}, \varepsilon\right) \cos \theta\right] .
\end{aligned}
$$

We shall study the flow of system (6) contained in the half-cylinder $\mathbb{R}^{+} \times \mathbb{S}^{1}=\{(r, \theta): r \geq 0$, $\theta \in(-\pi, \pi)\}$. Notice that after multiplying (6) by a power of $r$, the system can be extended for $r=0$. Therefore, the existence of a periodic orbit at infinity for system (4) is equivalent 
to the existence of the periodic orbit $r=0$ for system (6) on the cylinder. Now, consider the assumptions:

(a) the functions $R$ and $\xi$ are Lipschitz functions in the variable $r$ and they have period $2 \pi$ in the variable $\theta$.

(b) $R(0, \theta, \varepsilon)=0$ and $\xi(0, \theta, \varepsilon) \neq 0$ for all $\theta \in \mathbb{S}^{1}$ and for every $\varepsilon \geq 0$ sufficiently small.

Notice that $(a)$ and $(b)$ are sufficient and necessary conditions in order to guarantee that system (4) has a periodic solution at infinity. Finally, taking $\theta$ as the new independent variable the differential system (6) can be written as the first order differential equation

$$
r^{\prime}=\frac{d r}{d \theta}=S(r, \theta, \varepsilon)=\frac{R(r, \theta, \varepsilon)}{\xi(r, \theta, \varepsilon)} .
$$

Consequently, the Poincaré map defined on a neighborhood of $r=0$ is given by $\Pi(\rho)=$ $r(2 \pi, \rho, \varepsilon)$, where $r(\theta, \rho, \varepsilon)$ is the solution of $(7)$ such that $r(0, \rho, \varepsilon)=\rho$.

\section{Proof of the main Results}

In this section we provide the proofs of Proposition 1 and Theorems A, B and C.

3.1. Proof of Proposition 1. We assume that the piecewise linear vector field $W_{0}(x, y)$ satisfies the hypotheses $\left(H_{1}\right),\left(H_{2}\right)$ and $\left(H_{3}\right)$. Then, the left and right linear differential systems are written as $(\dot{x}, \dot{y})=M_{0}^{ \pm}(x, y)+\left(0, u_{2}^{ \pm}\right)^{T}$, where

$$
M_{0}^{ \pm}=\left(\begin{array}{cc}
m_{11}^{ \pm} & m_{12}^{ \pm} \\
m_{21}^{ \pm} & -m_{11}^{ \pm}
\end{array}\right)
$$

with $\left(m_{11}^{ \pm}\right)^{2}+m_{12}^{ \pm} m_{21}^{ \pm}<0$ and $m_{12}^{ \pm} \neq 0$.

Then, applying the change of variables $(\tilde{x}, \tilde{y})=\psi(x, y)=\left(x,-m_{11}^{-} x-m_{12}^{-} y\right)$ we obtain the following piecewise linear differential system

$$
\left(\begin{array}{c}
\tilde{x}^{\prime} \\
\tilde{y}^{\prime}
\end{array}\right)=\left\{\begin{array}{c}
\tilde{A}^{+}\left(\begin{array}{c}
\tilde{x} \\
\tilde{y}
\end{array}\right)+\left(\begin{array}{c}
0 \\
-m_{12}^{+} u_{2}^{+}
\end{array}\right) \quad \text { if } \quad \tilde{x} \geq 0 \\
\tilde{A}^{-}\left(\begin{array}{c}
\tilde{x} \\
\tilde{y}
\end{array}\right)+\left(\begin{array}{c}
0 \\
-m_{12}^{+} u_{2}^{-}
\end{array}\right) \quad \text { if } \quad \tilde{x} \leq 0
\end{array}\right.
$$

where

and

$$
\widetilde{A}^{+}=\left(\begin{array}{cc}
m_{11}^{+}-\frac{m_{11}^{-} m_{12}^{+}}{m_{12}^{-}} & -\frac{m_{12}^{+}}{m_{12}^{-}} \\
\frac{\left(m_{1}^{-}\right)^{2} m_{12}^{+}}{m_{12}^{-}}-2 m_{11}^{-} m_{11}^{+}-m_{12}^{-} m_{21}^{+} & \frac{m_{11}^{-} m_{12}^{+}}{m_{12}^{-}}-m_{11}^{+}
\end{array}\right)
$$

$$
\widetilde{A}^{-}=\left(\begin{array}{cc}
0 & -1 \\
-\left(m_{11}^{-}\right)^{2}-m_{12}^{-} m_{21}^{-} & 0
\end{array}\right) .
$$

Notice that the above change of variables fixes the switching manifold. 
Now, let $\rho=\sqrt{\left|\left(m_{11}^{-}\right)^{2}+m_{12}^{-} m_{21}^{-}\right|}$, where $\left(m_{11}^{-}\right)^{2}+m_{12}^{-} m_{21}^{-}<0$. Proceeding with the following change of variables and rescaling of time

$$
(\tilde{x}, \tilde{y}, \tilde{t}) \mapsto\left(\frac{x}{\rho}, y, \frac{t}{\rho}\right)
$$

system (8) becomes

$$
Z_{0}^{-}(x, \tilde{y})=\left(\begin{array}{cc}
0 & -1 \\
1 & 0
\end{array}\right)\left(\begin{array}{l}
x \\
\tilde{y}
\end{array}\right)+\left(\begin{array}{l}
0 \\
e
\end{array}\right), \quad \text { for } \quad x \leq 0
$$

and

$$
Z_{0}^{+}(x, \tilde{y})=\left(\begin{array}{cc}
a & b \\
c & -a
\end{array}\right)\left(\begin{array}{l}
x \\
\tilde{y}
\end{array}\right)+\left(\begin{array}{l}
0 \\
d
\end{array}\right), \quad \text { for } \quad x \geq 0
$$

where

$$
\begin{aligned}
& a=\frac{1}{\rho}\left(m_{11}^{+}-\frac{m_{11}^{-} m_{12}^{+}}{m_{12}^{-}}\right), \\
& b=-\frac{m_{12}^{+}}{m_{12}^{-}} \\
& c=\frac{1}{\rho^{2}}\left(\frac{\left(m_{11}^{-}\right)^{2} m_{12}^{+}}{m_{12}^{-}}-2 m_{11}^{-} m_{11}^{+}-m_{12}^{+} m_{21}^{+}\right), \\
& d=-\frac{m_{12}^{+} u_{2}^{+}}{\rho} \text { and } \\
& e=-\frac{m_{12}^{-} u_{2}^{-}}{\rho}>0 .
\end{aligned}
$$

The singular points of $Z_{0}^{-}, Z_{0}^{+}$are given by $p^{-}=(-e, 0)$ and $p^{+}=\frac{d}{a^{2}+b c}(-b, a)$, resp.. From $\left(H_{1}\right),\left(H_{2}\right)$ and $\left(H_{3}\right)$ we conclude that $b<0, c>0, d>0, e>0$ and $a^{2}+b c<0$.

3.2. Study of the infinity. Applying the Bendixson change of coordinates given in (2) to $Z_{\varepsilon}$, we obtain that the differential system in $\Sigma^{-}$can be written

$$
\begin{aligned}
\frac{d u^{-}}{d t}= & -v(2 e u+1)\left(u^{2}+v^{2}\right)+\varepsilon\left(-u^{3}\left(v_{1}^{-} u+b_{11}^{-}\right)+v_{1}^{-} v^{4}+u v^{2}\left(b_{11}^{-}-2 b_{22}^{-}\right)\right. \\
& \left.-u^{2} v\left(a_{2}+2\left(v_{2}^{-} u+b_{21}^{-}\right)\right)+v^{3}\left(a_{2}-2 v_{2}^{-} u\right)\right)\left.\right|_{u=u^{-}, v=v^{-}} \\
\frac{d v^{-}}{d t}= & e u^{4}-e v^{4}+u^{3}+u v^{2}+\varepsilon\left(u^{2} v\left(-2 v_{1}^{-} u-2 b_{11}^{-}+b_{22}^{-}\right)-v^{3}\left(2 v_{1}^{-} u+b_{22}^{-}\right)\right. \\
& \left.-u v^{2}\left(2 a_{2}+b_{21}^{-}\right)+u^{3}\left(v_{2}^{-} u+b_{21}^{-}\right)-v_{2}^{-} v^{4}\right)\left.\right|_{u=u^{-}, v=v^{-}}
\end{aligned}
$$


and for the differential system in $\Sigma^{+}$can be written as

$$
\begin{aligned}
\frac{d u^{+}}{d t}= & -a u^{3}+3 a u v^{2}-u^{2} v(b+2(c+d u))+v^{3}(b-2 d u)+\varepsilon\left(-u^{3}\left(v_{1}^{+} u+b_{11}^{+}\right)\right. \\
& \left.+v_{1}^{+} v^{4}+u v^{2}\left(b_{11}^{+}-2 b_{22}^{+}\right)-u^{2} v\left(c_{2}+2\left(d_{0} u+d_{1}\right)\right)+v^{3}\left(c_{2}-2 d_{0} u\right)\right)\left.\right|_{u=u^{+}, v=v^{+}}, \\
\frac{d v^{+}}{d t}= & -3 a u^{2} v+a v^{3}-u v^{2}(2 b+c)+u^{3}(c+d u)+\varepsilon\left(u^{2} v\left(-2 v_{1}^{+} u-2 b_{11}^{+}+b_{22}^{+}\right)\right. \\
& \left.-v^{3}\left(2 v_{1}^{+} u+b_{22}^{+}\right)-u v^{2}\left(2 c_{2}+d_{1}\right)+u^{3}\left(d_{0} u+d_{1}\right)-d_{0} v^{4}\right)-\left.d v^{4}\right|_{u=u^{+}, v=v^{+}} .
\end{aligned}
$$

Applying the polar change of coordinates the left differential system can be written as

$$
\begin{aligned}
\frac{d r^{-}}{d t}= & -e r^{2} \sin \theta-r \varepsilon\left(\cos \theta\left(v_{1}^{-} r+\left(a_{2}+b_{21}^{-}\right) \sin \theta\right)+b_{11}^{-} \cos ^{2} \theta\right. \\
& \left.+\sin \theta\left(v_{2}^{-} r+b_{22}^{-} \sin \theta\right)\right)\left.\right|_{r=r^{-}, \theta=\theta^{-}}, \\
\frac{d \theta^{-}}{d t}= & 1+e r \cos \theta+\varepsilon\left(-\sin \theta\left(v_{1}^{-} r+a_{2} \sin \theta\right)+\cos \theta\left(\left(b_{22}^{-}-b_{11}^{-}\right) \sin \theta\right.\right. \\
& \left.\left.+v_{2}^{-} r\right)+b_{21}^{-} \cos ^{2} \theta\right)\left.\right|_{r=r^{-}, \theta=\theta^{-}},
\end{aligned}
$$

and, similarly, the right differential system can be written as

$$
\begin{aligned}
\frac{d r^{+}}{d t}= & -r(a \cos (2 \theta)+\sin \theta((b+c) \cos \theta+d r))-r \varepsilon\left(\operatorname { c o s } \theta \left(v_{1}^{+} r\right.\right. \\
& \left.\left.+\left(c_{2}+d_{1}\right) \sin \theta\right)+b_{11}^{+} \cos ^{2} \theta+\sin \theta\left(d_{0} r+b_{22}^{+} \sin \theta\right)\right)\left.\right|_{r=r^{+}, \theta=\theta^{+}}, \\
\frac{d \theta^{+}}{d t}= & \cos \theta(d r-2 a \sin \theta)-b \sin ^{2} \theta+c \cos ^{2} \theta+\varepsilon\left(-\sin \theta\left(v_{1}^{+} r+c_{2} \sin \theta\right)\right. \\
& \left.+\cos \theta\left(\left(b_{22}^{+}-b_{11}^{+}\right) \sin \theta+d_{0} r\right)+d_{1} \cos ^{2} \theta\right)\left.\right|_{r=r^{+}, \theta=\theta^{+}} .
\end{aligned}
$$

Considering the rescaling of the radius given by $r^{ \pm} t=\varepsilon^{3} \rho^{ \pm} t$ and taking $\theta^{-}$as the new independent variable we obtain

$$
\begin{aligned}
\frac{d \rho^{-}}{d \theta^{-}}= & -\varepsilon \rho\left(b_{11}^{-} \cos ^{2} \theta+\left(a_{2}+b_{21}^{-}\right) \sin \theta \cos \theta+b_{22}^{-} \sin ^{2}(\theta)\right) \\
& +\varepsilon^{2} \rho\left(\left(b_{22}^{-}-b_{11}^{-}\right) \sin \theta \cos \theta-a_{2} \sin ^{2} \theta+b_{21}^{-} \cos ^{2} \theta\right) \\
& \left(b_{11}^{-} \cos ^{2} \theta+\left(a_{2}+b_{21}^{-}\right) \sin \theta \cos \theta+b_{22}^{-} \sin ^{2} \theta\right)+\left.\mathcal{O}\left(\varepsilon^{3}\right)\right|_{\rho=\rho^{-}, \theta=\theta^{-}},
\end{aligned}
$$

and

$$
\begin{aligned}
\frac{d \rho^{+}}{d \theta^{+}}= & \frac{\rho(2 a \cos (2 \theta)+(b+c) \sin (2 \theta))}{2\left(a \sin (2 \theta)+b \sin ^{2} \theta-c \cos ^{2} \theta\right)}-\varepsilon \frac{\rho}{2\left(a \sin (2 \theta)+b \sin ^{2} \theta-c \cos ^{2} \theta\right)^{2}} \\
& \left(-\sin (2 \theta)\left(a\left(b_{11}^{+}+b_{22}^{+}\right)+b d_{1}-c c_{2}\right)+\cos (2 \theta)\left(a c_{2}-a d_{1}+b b_{22}^{+}+c b_{11}^{+}\right)\right. \\
& \left.-a c_{2}-a d_{1}-b b_{22}^{+}+c b_{11}^{+}\right)+\left.\mathcal{O}\left(\varepsilon^{2}\right)\right|_{\rho=\rho^{+}, \theta=\theta^{+}} .
\end{aligned}
$$


Let $\rho^{\mp}(\theta)=\rho_{0}^{\mp}(\theta)+\varepsilon \rho_{1}^{\mp}(\theta)+\mathcal{O}\left(\varepsilon^{2}\right)$ be the solution of $\frac{d \rho^{\mp}}{d \theta^{\mp}}$ satisfying the initial condition $\rho^{ \pm}\left(-\frac{\pi}{2}\right)=\rho_{0}$. So, we have

$$
\begin{aligned}
\rho_{0}^{-}(\theta)= & \rho_{0} \\
\rho_{0}^{+}(\theta)= & \frac{\rho_{0}}{\sqrt{2}}\left(\sqrt{\frac{2 a \sin (2 \theta)-(b+c) \cos (2 \theta)+b-c}{b}}\right) \\
\rho_{1}^{-}(\theta)= & \frac{\rho_{0}}{4}\left(-2 b_{11}^{-} \theta-b_{11}^{-} \sin (2 \theta)+\pi b_{11}^{-}+a_{2} \cos (2 \theta)+a_{2}+b_{21}^{-} \cos (2 \theta)+b_{21}^{-}-2 b_{22}^{-} \theta\right. \\
& \left.+b_{22}^{-} \sin (2 \theta)+\pi b_{22}^{-}\right), \\
& +\frac{\rho_{0}}{4 b \xi \sqrt{-2 b} \sqrt{-2 a \sin (2 \theta)+(b+c) \cos (2 \theta)-b+c}}\left(2 b ( b _ { 1 1 } ^ { + } + b _ { 2 2 } ^ { + } ) \operatorname { a r c t a n } \left(\frac{a}{\xi}\right.\right. \\
& \left.+2 a c_{2} \xi+b \xi\left(b_{22}^{+}-b_{11}^{+}\right)\right)-\cos (2 \theta)\left(\pi b(b+c)\left(b_{11}^{+}+b_{22}^{+}\right)+2 \xi\left(c c_{2}-b d_{1}\right)\right) \\
& \left.+\pi b(b-c)\left(b_{11}^{+}+b_{22}^{+}\right)+2 b d_{1} \xi-2 c c_{2} \xi\right)
\end{aligned}
$$

where $a^{2}+b c=-\xi^{2}$, with $\xi>0$. Therefore, the displacement map writes

$$
\begin{aligned}
\rho\left(\rho_{0}\right) & =\rho_{0}^{+}(\pi / 2)-\rho_{0}^{-}(-3 \pi / 2)+\varepsilon\left(\rho_{1}^{+}(\pi / 2)-\rho_{1}^{-}(-3 \pi / 2)\right)+\mathcal{O}\left(\varepsilon^{2}\right) \\
& =-\frac{1}{2} \varepsilon \pi \rho_{0}\left(b_{11}^{-}+b_{22}^{-}+\frac{b_{11}^{+}+b_{22}^{+}}{\xi}\right)+\mathcal{O}\left(\varepsilon^{2}\right) .
\end{aligned}
$$

Consequently, if $\xi\left(b_{11}^{-}+b_{22}^{-}\right)+b_{11}^{+}+b_{22}^{+}>0\left(\right.$ resp. $\left.\xi\left(b_{11}^{-}+b_{22}^{-}\right)+b_{11}^{+}+b_{22}^{+}<0\right)$, then the infinity is a stable (resp. unstable) periodic solution.

3.3. Proof of Theorem A. The proof will be split in three steps. In the first one we prove that the number of crossing limit cycles of $Z_{1, \varepsilon}(X)$ is given by the zeros of the first order Melnikov function

$$
\begin{aligned}
M_{1}\left(y_{0}\right)= & \frac{1}{2 y_{0}}\left(4 v_{1}^{-} y_{0}-2\left(b_{11}^{-}+b_{22}^{-}\right)\left(\pi\left(e^{2}+y_{0}^{2}\right)+e y_{0}\right)\right. \\
& +\left(b_{11}^{-}+b_{22}^{-}\right)\left(e^{2}+y_{0}^{2}\right) \arccos \left(\frac{2 e^{2}}{e^{2}+y_{0}^{2}}-1\right) \\
& -\frac{1}{b \xi^{3}}\left(-2 b d y_{0} \xi\left(b_{11}^{+}+b_{22}^{+}\right)-4 v_{1}^{+} y_{0} \xi^{3}+b\left(b_{11}^{+}+b_{22}^{+}\right)\left(d^{2}+y_{0}^{2} \xi^{2}\right)\right. \\
& \left.\left.\arccos \left(\frac{2 d^{2}}{d^{2}+y_{0}^{2} \xi^{2}}-1\right)\right)\right),
\end{aligned}
$$

were the Melnikov Function is given by

$$
M\left(y_{0}, \varepsilon\right)=M_{0}\left(y_{0}\right)+M_{1}\left(y_{0}\right) \varepsilon+M_{2}\left(y_{0}\right) \varepsilon^{2}+\mathcal{O}(\varepsilon)^{3}
$$

and $M_{i}\left(y_{0}\right)=M_{i}^{-}\left(y_{0}\right)-M_{i}^{+}\left(y_{0}\right)$ for $i=0,1,2$. 
In the second one we prove that the upper bound of the number of zeros is three, and that this number is reached. Finally, in the third step we study the stability of the crossing limit cycles.

Lemma 4. The zeros of $M_{1}\left(y_{0}\right)$ correspond to crossing limit cycles for $Z_{1, \varepsilon}(X)$.

Proof. Let $Z_{\varepsilon}$ be given by (2). From hypotheses we know that $b<0, d>0$ and $a^{2}+b c<0$. So, denote $a^{2}+b c=-\xi^{2}, \xi>0$. Let $\left(x_{\varepsilon}^{ \pm}(t), y_{\varepsilon}^{ \pm}(t)\right)$ be the trajectories of the linear vector fields $Z_{0}^{ \pm}$satisfying $x_{\varepsilon}^{-}(0)=0, y_{\varepsilon}^{-}(0)=y_{0}>0$, and $x_{\varepsilon}^{+}(0)=0, y_{\varepsilon}^{+}(0)=y_{1}$. So, for $\varepsilon=0$, we compute

$$
\begin{aligned}
& x_{0}^{-}(t)=e(-1+\cos t)-y_{0} \sin t, \\
& y_{0}^{-}(t)=y_{0} \cos t+e \sin t
\end{aligned}
$$

and

$$
\begin{aligned}
& x_{0}^{+}(s)=\frac{b\left(d-d \cos (s \xi)+y_{1} \xi \sin (s \xi)\right)}{\xi^{2}}, \\
& y_{0}^{+}(s)=\frac{1}{\xi^{2}}\left(-d+\left(d+y_{1} \xi^{2}\right) \cos (s \xi)+\xi\left(d-y_{1}\right) \sin (s \xi)\right) .
\end{aligned}
$$

Let $t_{l, \varepsilon}>0$ and $t_{r, \varepsilon}<0$ be the first return times to $\Sigma$ of the above solutions, that is $x_{\varepsilon}^{-}\left(t_{l}\right)=x_{\varepsilon}^{+}\left(t_{r}\right)=0$. For $\varepsilon=0$ we have

$$
t_{l 0}=2 \pi-\arccos \left(\frac{2 e^{2}}{e^{2}+y_{0}^{2}}-1\right)
$$

and

$$
t_{r 0}=-\frac{1}{\xi} \arccos \left(\frac{2 d^{2}}{d^{2}+\xi^{2} y_{1}^{2}}-1\right) .
$$

Writing $t_{l, \varepsilon}=t_{l 0}+t_{l 1} \varepsilon+\mathcal{O}\left(\varepsilon^{2}\right)$ and $t_{r, \varepsilon}=t_{r 0}+t_{r 1} \varepsilon+\mathcal{O}\left(\varepsilon^{2}\right)$, the coefficients $t_{l 1}$ and $t_{r 1}$ can be computed by expanding the equations $x_{\varepsilon}^{-}\left(t_{l}\right)=0$ and $x_{\varepsilon}^{+}\left(t_{r}\right)=0$ around $\varepsilon=0$. So

$$
\begin{aligned}
t_{l 1}= & \frac{1}{2 y_{0}\left(e^{2}+y_{0}^{2}\right)}\left(2 y_{0}\left(2 v_{1}^{-} e-e\left(e\left(b_{11}^{-}+b_{22}^{-}\right)+y_{0}\left(b_{21}^{-}-a_{2}\right)\right)+2 v_{2}^{-} y_{0}\right)-2 \pi\left(e^{2}+y_{0}^{2}\right)\left(e \left(b_{11}^{-}\right.\right.\right. \\
& \left.\left.\left.+b_{22}^{-}\right)+y_{0}\left(b_{21}^{-}-a_{2}\right)\right)+\left(e^{2}+y_{0}^{2}\right) \arccos \left(\frac{2 e^{2}}{e^{2}+y_{0}^{2}}-1\right)\left(e\left(b_{11}^{-}+b_{22}^{-}\right)+y_{0}\left(b_{21}^{-}-a_{2}\right)\right)\right),
\end{aligned}
$$

and

$$
\begin{aligned}
t_{r 1}= & \frac{1}{2 b y_{1} \xi^{3}\left(d^{2}+y_{1}^{2} \xi^{2}\right)}\left(-2 d y_{1} \xi\left(y_{1}\left(a^{2} c_{2}+a b\left(b_{22}^{+}-b_{11}^{+}\right)-b^{2} d_{1}\right)+b d\left(b_{11}^{+}+b_{22}^{+}\right)\right)\right. \\
& +2 y_{1} \xi^{3}\left(2 a v_{1}^{+} y_{1}+2 b d_{0} y_{1}-2 v_{1}^{+} d-c_{2} d y_{1}\right)+\left(c_{2} y_{1}\left(a^{2}+\xi^{2}\right)+b\left(-a b_{11}^{+} y_{1}+a b_{22}^{+} y_{1}\right.\right. \\
& \left.\left.\left.+b_{11}^{+} d+d b_{22}^{+}\right)-b^{2} d_{1} y_{1}\right)\left(d^{2}+y_{1}^{2} \xi^{2}\right) \arccos \left(\frac{2 d^{2}}{d^{2}+y_{1}^{2} \xi^{2}}-1\right)\right) .
\end{aligned}
$$

Replacing the expression of $t_{l 0}$ and $t_{l 1}$ in the expansion of the solution of (10) we get the positive half return map in $\Sigma^{-}$, i.e.

$$
M_{1}^{-}=\frac{4 v_{1}^{-} y_{0}-2\left(b_{11}^{-}+b_{22}^{-}\right)\left(\pi e^{2}+e y_{0}+\pi y_{0}^{2}\right)+\left(b_{11}^{-}+b_{22}^{-}\right)\left(e^{2}+y_{0}^{2}\right) \arccos \left(\frac{2 e^{2}}{e^{2}+y_{0}^{2}}-1\right)}{2 y_{0}} .
$$


Analogously, replacing the expressions of $t_{r 0}$ and $t_{r 1}$ in the expansion of the solution of (11) we obtain the negative half return map in $\Sigma^{+}$, namely

$$
M_{1}^{+}=\frac{b\left(b_{11}^{+}+b_{22}^{+}\right)\left(d^{2}+\xi^{2} y_{1}^{2}\right) \arccos \left(\frac{2 d^{2}}{d^{2}+\xi^{2} y_{1}^{2}}-1\right)-2 b d \xi y_{1}\left(b_{11}^{+}+b_{22}^{+}\right)-4 v_{1}^{+} \xi^{3} y_{1}}{2 b \xi^{3} y_{1}} .
$$

The difference between $M_{1}^{-}$and $M_{1}^{+}$provides the first order Melnikov function $M_{1}\left(y_{0}\right)=$ $M_{1}^{-}\left(y_{0}\right)-M_{1}^{+}\left(y_{0}\right)$ given in (9), and the simple zeros of $M_{1}\left(y_{0}\right)$ provide the crossing limit cycles of $Z_{1, \varepsilon}(X)$.

Lemma 5. The function $M_{1}$ presented in (9) has at most three simple zeros. Furthermore, this upper bound is reached.

Proof. Considering the change of coordinates and parameters given by $y_{0}=\alpha s_{0} / \xi, e=\alpha / \xi$ and $d=\alpha \beta$ in the function $M_{1}$, given in (9), we obtain

$$
\begin{aligned}
M_{1}\left(s_{0}\right)= & -\frac{1}{2 b \beta s_{0} \xi^{2}}\left(-4 \beta s_{0} \xi^{2}\left(v_{1}^{-} b+v_{1}^{+}\right)-2 \alpha b \beta^{2} s_{0}\left(b_{11}^{+}+b_{22}^{+}\right)+\alpha b \xi\left(b_{11}^{-}+b_{22}^{-}\right)\left(\pi \beta^{2} s_{0}^{2}\right.\right. \\
& \left.+2 \beta s_{0}+\pi\right)+\alpha b \xi\left(b_{11}^{-}+b_{22}^{-}\right)\left(\beta^{2} s_{0}^{2}+1\right) \arccos \left(1-\frac{2}{\beta^{2} s_{0}^{2}+1}\right)+\alpha b \beta^{2}\left(s_{0}^{2}+1\right) \\
& \left.\left(b_{11}^{+}+b_{22}^{+}\right) \arccos \left(\frac{2}{s_{0}^{2}+1}-1\right)\right) .
\end{aligned}
$$

The positive zeros of $M_{1}\left(s_{0}\right)$ coincide with the zeros of $2 b \beta s_{0} \xi^{2} M_{1}\left(s_{0}\right)=\widetilde{M}_{1}\left(s_{0}\right)$. We have that

$$
\begin{aligned}
\widetilde{M}_{1}\left(s_{0}\right)= & 2 \alpha b \beta^{2} s_{0}\left(b_{11}^{+}+b_{22}^{+}\right)+4 \beta s_{0} \xi^{2}\left(v_{1}^{-} b+v_{1}^{+}\right)-\alpha b \xi\left(b_{11}^{-}+b_{22}^{-}\right)\left(\pi \beta^{2} s_{0}^{2}+2 \beta s_{0}+\pi\right) \\
& -\alpha b \xi\left(b_{11}^{-}+b_{22}^{-}\right)\left(\beta^{2} s_{0}^{2}+1\right) \arccos \left(1-\frac{2}{\beta^{2} s_{0}^{2}+1}\right)-\alpha b \beta^{2}\left(s_{0}^{2}+1\right)\left(b_{11}^{+}+b_{22}^{+}\right) \\
& \arccos \left(\frac{2}{s_{0}^{2}+1}-1\right) .
\end{aligned}
$$

If

$$
\begin{aligned}
& K_{0}=2 \beta\left(2 \xi^{2}\left(v_{1}^{-} b+v_{1}^{+}\right)-\alpha b \xi\left(b_{11}^{-}+b_{22}^{-}\right)+\alpha b \beta\left(b_{11}^{+}+b_{22}^{+}\right)\right), \\
& K_{1}=\alpha b \xi\left(b_{11}^{-}+b_{22}^{-}\right), \\
& K_{2}=-b \alpha \beta^{2}\left(b_{11}^{+}+b_{22}^{+}\right),
\end{aligned}
$$

then $\widetilde{M}_{1}$ can be rewritten as

$$
\widetilde{M}_{1}\left(s_{0}\right)=K_{0} s_{0}+K_{1}\left(\beta^{2} s_{0}^{2}+1\right)\left(\arccos \left(\frac{2}{\beta^{2} s_{0}^{2}+1}-1\right)-2 \pi\right)+K_{2}\left(s_{0}^{2}+1\right) \arccos \left(\frac{2}{s_{0}^{2}+1}-1\right) .
$$

Note that $\widetilde{M}_{1}\left(s_{0}\right)$ is the linear combination

$$
\widetilde{M}_{1}\left(s_{0}\right)=K_{0} f_{0}\left(s_{0}\right)-2 \pi K_{1} f_{1}\left(s_{0}\right)+K_{2} f_{2}\left(s_{0}\right)+K_{1} f_{3}\left(s_{0}\right)
$$


of the functions

$$
\begin{aligned}
& f_{0}\left(s_{0}\right)=g_{0} \\
& f_{1}\left(s_{0}\right)=1+\beta^{2} s_{0}^{2} \\
& f_{2}\left(s_{0}\right)=\left(s_{0}^{2}+1\right) \arccos \left(\frac{2}{s_{0}^{2}+1}-1\right) \\
& f_{3}\left(s_{0}\right)=\left(\beta^{2} s_{0}^{2}+1\right) \arccos \left(\frac{2}{\beta^{2} s_{0}^{2}+1}-1\right) .
\end{aligned}
$$

Denoting $W_{k}\left(s_{0}\right)=W_{k}\left(f_{0}, f_{1}, \ldots, f_{k}\right)\left(s_{0}\right)$, we have

$$
\begin{aligned}
& W_{0}\left(s_{0}\right)=s_{0} \\
& W_{1}\left(s_{0}\right)=\beta^{2} s_{0}^{2}-1 \\
& W_{2}\left(s_{0}\right)=2\left(\beta^{2}-1\right) \arccos \left(\frac{2}{s_{0}^{2}+1}-1\right)-\frac{4\left(\beta^{2}+1\right) s_{0}}{s_{0}^{2}+1} \\
& W_{3}\left(s_{0}\right)=\frac{16 \beta^{3}\left(\beta^{2}-1\right)\left(2 s_{0}\left(s_{0}^{2}-1\right)+\left(s_{0}^{2}+1\right)^{2} \arccos \left(\frac{2}{s_{0}^{2}+1}-1\right)\right)}{\left(s_{0}^{2}+1\right)^{2}\left(\beta^{2} s_{0}^{2}+1\right)^{2}} .
\end{aligned}
$$

Observe that the functions $W_{k}\left(s_{0}\right)$ for $k=0,1,2,3$ have not roots if $s_{0}>1 / \beta$. In fact, we have that

$$
W_{3}\left(s_{0}\right)\left(\beta^{2} s_{0}^{2}+1\right)^{2}=\widetilde{W}_{3}\left(s_{0}\right)
$$

where

$$
\widetilde{W}_{3}\left(s_{0}\right)=\frac{16 \beta^{3}\left(\beta^{2}-1\right)\left(2 s_{0}\left(s_{0}^{2}-1\right)+\left(s_{0}^{2}+1\right)^{2} \arccos \left(\frac{2}{s_{0}^{2}+1}-1\right)\right)}{\left(s_{0}^{2}+1\right)^{2}} .
$$

Computing the derivative of $\widetilde{W}_{3}\left(s_{0}\right)$ we have

$$
\frac{d \widetilde{W_{3}}}{d s_{0}}=\frac{256 \beta^{3}\left(\beta^{2}-1\right) s_{0}^{2}}{\left(s_{0}^{2}+1\right)^{3}}
$$

which is strictly positive for all $s_{0} \neq 0$ and $\beta>1$, and strictly negative for all $s_{0} \neq 0$ and $0<\beta<1$. Therefore, since $\lim _{s_{0} \rightarrow 0} \widetilde{W_{3}}\left(s_{0}\right)=0$ and $\lim _{s_{0} \rightarrow \infty} \widetilde{W}_{3}\left(s_{0}\right)=0$, the function $\widetilde{W}_{3}$ has no roots for $s_{0}>0$. Hence, the function $W_{3}$ has no roots if $s_{0}>0$ and $\beta \neq 1$.

In summary, the ordered set $\mathcal{F}=\left[f_{0}, f_{1}, f_{2}, f_{3}\right]$ is an ET-Chebyschev System. By Theorem 2 we conclude that there exists a linear combination of the functions of $\mathcal{F}$ with at most three roots. So, the upper bound for the number of zeros of any function in the linear space of functions generated by the functions of $\mathcal{F}$ is three. In Example 4.1 of Section 4 we show that this upper bound for the zeros is reached.

Lemma 6. The highest amplitude limit cycle (when it exists) is stable (resp. unstable) provided that $\xi\left(b_{11}^{-}+b_{22}^{-}\right)+b_{11}^{+}+b_{22}^{+}<0$ (resp. $\left.\xi\left(b_{11}^{-}+b_{22}^{-}\right)+b_{11}^{+}+b_{22}^{+}>0\right)$. The lowest amplitude limit cycle (when it exists) is stable (resp. unstable) provided that $b_{11}^{-}+b_{22}^{-}<0$ or $b_{11}^{-}+b_{22}^{-}=0$ and $b v_{1}^{-}+v_{1}^{+}>0\left(\right.$ resp. $b_{11}^{-}+b_{22}^{-}>0$ or $b_{11}^{-}+b_{22}^{-}=0$ and $\left.b v_{1}^{-}+v_{1}^{+}<0\right)$. 
Proof. In what follows we study the stability of the crossing limit cycles. In fact, this stability depends of the sign of $M_{1}$. Indeed, $M_{1}\left(y_{0}\right)=M_{1}^{-}\left(y_{0}\right)-M_{1}^{+}\left(y_{0}\right)$, let $y_{0}^{*} \in \mathbb{R}^{+}$such that $M_{1}\left(y_{0}\right)=0$, if $M_{1}\left(y_{0}\right)>0$ for $y_{0}<y_{0}^{*}$ and $M_{1}\left(y_{0}\right)<0$ for $y_{0}>y_{0}^{*}$, then the crossing limit cycle defined by $y_{0}$ is unstable. If these signs are reversed then it is stable.

We get that

$$
\lim _{s_{0} \rightarrow 0} \widetilde{M}_{1}\left(s_{0}\right)=-2 \pi \alpha b \xi\left(b_{11}^{-}+b_{22}^{-}\right), \text {and } \lim _{s_{0} \rightarrow \infty} M_{1}\left(s_{0}\right)=\xi\left(b_{11}^{-}+b_{22}^{-}\right)+b_{11}^{+}+b_{22}^{+} .
$$

Therefore, since $b<0, \alpha>0$ and $\xi>0$ the $\operatorname{sign}\left(\lim _{s_{0} \rightarrow 0} \widetilde{M}_{1}\left(s_{0}\right)\right)=\operatorname{sign}\left(b_{11}^{-}+b_{22}^{-}\right)$, and $2 b \beta s_{0} \xi^{2} m_{11}^{-}\left(s_{0}\right)=\widetilde{M}_{1}\left(s_{0}\right)$ so $\operatorname{sign}\left(\lim _{s_{0} \rightarrow 0} M_{1}\left(s_{0}\right)\right)=-\operatorname{sign}\left(b_{11}^{-}+b_{22}^{-}\right)$, see Figure 4 . So, the lemma follows.
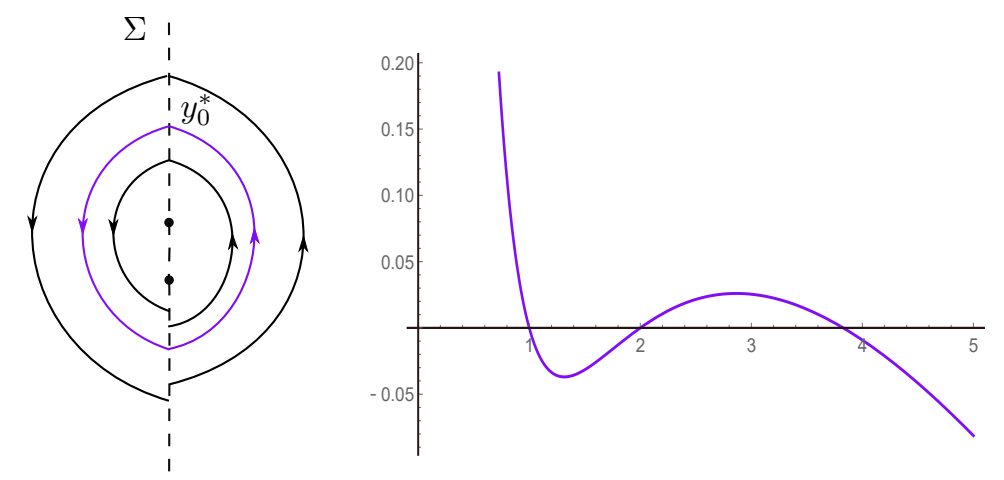

Figure 4. The repealer crossing limit cycle and the graphic of $M_{1}\left(y_{0}\right)$ 1-order linear perturbation of a piecewise linear center.

Now, we complete the proof of Theorem A analyzing the stability of the crossing limit cycles and of the periodic orbit at infinity. In fact, the stability of the periodic orbit at infinity is given by $\operatorname{sign}\left(\xi\left(b_{11}^{-}+b_{22}^{-}\right)+b_{11}^{+}+b_{22}^{+}\right)$, see Section 3.2 , and the stability of the crossing limit cycle (c.l.c.) of the biggest amplitude limit cycle is given in Table 1.

\begin{tabular}{|c|c|c|}
\hline $\operatorname{sign}\left(\xi\left(b_{11}^{-}+b_{22}^{-}\right)+b_{11}^{+}+b_{22}^{+}\right)$ & stability of the bigger c.l.c & stability of $\infty$ \\
\hline \hline-1 & stable & unstable \\
\hline 1 & unstable & stable \\
\hline
\end{tabular}

TABLE 1. Stability of the highest amplitude crossing limit cycle and the infinity.

3.4. Proof of Theorem B. In order to study the sliding/escaping limit cycle we consider the second order linear perturbation of the vector field $Z$ given in (2). The singular points and the spectrum of the systems in $\Sigma^{-}$and $\Sigma^{+}$are given by $p^{-}=(-e, 0)$ and $p^{+}=$ $\left(-b d /\left(a^{2}+b c\right),-d /\left(a^{2}+b c\right)\right)$, respectively; and the eigenvalues of the unperturbed piecewise linear vector field $Z_{2,0}(X)$ in $\Sigma^{-}$and $\Sigma^{+}$are given by $\operatorname{Spec}^{-}=\{i,-i\}$ and $S p e c^{+}=$ 
$\left\{-\sqrt{a^{2}+b c}, \sqrt{a^{2}+b c}\right\}$, respectively. Consider $Z_{\varepsilon}$ given in (2) and assume that $b_{11}^{-}=-b_{22}^{-}$, the fold point in $\Sigma^{-}$and $\Sigma^{+}$are given by

$$
\begin{aligned}
& y_{f 1}=v_{1}^{-} \varepsilon+\left(A_{0}+v_{1}^{-} a_{2}\right) \varepsilon^{2}+\mathcal{O}\left(\varepsilon^{3}\right) \text { and } \\
& y_{f 2}=-\frac{v_{1}^{+} \varepsilon}{b}+\frac{\left(-b C_{0}+v_{1}^{+} c_{2}\right) \varepsilon^{2}}{b^{2}}+\mathcal{O}\left(\varepsilon^{3}\right),
\end{aligned}
$$

respectively. Under the assumptions $e>0$ and $d b<0$ we get that $y_{f 1}$ is a visible fold point and $y_{f 2}$ is an invisible fold point. Assuming that $v_{1}^{-}>-\frac{v_{1}^{+}}{b}$ we have that $y_{f 1}$ is over of $y_{f 2}$ and by (3) the expression of the sliding vector fields is

$$
\begin{aligned}
Z_{\varepsilon}^{s}(0, y)= & y(a y-b e-d)+\varepsilon\left(v_{1}^{-}(d-a y)-y\left(y\left(a a_{2}+b b_{22}^{-}+b_{22}^{+}\right)-a_{2} d+b v_{2}^{-}+c_{2} e+d_{0}\right)\right. \\
& \left.-v_{1}^{+} e\right)+\varepsilon^{2}\left(-y\left(a A_{0}-v_{1}^{-} b_{22}^{+}-a_{2} d_{0}-A_{2} d+b w_{2}^{-}+v_{2}^{-} c_{2}+b_{22}^{-} v_{1}^{+}+C_{2} e\right.\right. \\
& \left.\left.+D_{0}\right)+y^{2}\left(-\left(a A_{2}-a_{2} b_{22}^{+}+b c_{22}^{-}+b_{22}^{-} c_{2}+D_{2}\right)\right)+v_{1}^{-} d_{0}+A_{0} d-v_{2}^{-} v_{1}^{+}-C_{0} e\right)
\end{aligned}
$$

Any point in the sliding region is given as a convex combination of $y_{f 1}$ and $y_{f 2}$ as follows

$$
y_{s}(\lambda)=(1-\lambda) y_{f 1}+\lambda y_{f 2}=\varepsilon\left(v_{1}^{-}-v_{1}^{-} \lambda-\frac{v_{1}^{+} \lambda}{b}\right)
$$

where $0<\lambda<1$. A necessary condition for the existence of a sliding/escaping limit cycle is that the sliding vector field is regular and points toward the visible fold point $y_{f 1}$. The pseudo-equilibrium is $\left(0, y^{*}\right)$ with $y^{*}=(d+b e) / a$, which is reached when $\lambda=\lambda^{*}=$ $-b(d+b e) /\left(a\left(v_{1}^{-} b+v_{1}^{+}\right) \varepsilon\right)$. Under the hypotheses $a<0$ and $d+b e>0$, we obtain that $\left(0, y^{*}\right) \notin \Sigma^{s}$, i.e., $Z_{\varepsilon}^{s}$ is regular. The direction of the sliding vector field is given by the sign of the derivative of $Z_{\varepsilon}^{s}$ evaluated at $y_{s}$, that is by $\left(v_{1}^{-} b+v_{1}^{+}\right)(b e+d) / b$. From assumptions $(d+b e)>0$ and $\left(v_{1}^{-} b+v_{1}^{+}\right)<0\left(\left(v_{1}^{-} b+v_{1}^{+}\right)>0\right.$ resp. $)$, we conclude that the sliding (escaping resp.) vector field points towards $y_{f 1}$ ( $y_{f 2}$ resp.).

In what follows we study the return maps passing through the fold point of $Z_{\varepsilon}^{+}$and $Z_{\varepsilon}^{-}$. Our goal is to provide an order relation between the images by the flow of the fold points in a transverse section through $y_{f 1}$. This analysis not only provides a necessary condition for the existence of a sliding/escaping limit cycle, but also provides its distinct topological type. The negative half return map in a neighborhood of the invisible fold point $y_{f 2}$ defines the involution $\gamma_{Z_{\varepsilon}^{+}}: I^{-} \rightarrow I^{+}$, where $I^{+}, I^{-}$is an open interval above, below, resp., of $y_{f 2}$. For more details about the construction of this involution, see [19]. In this way, we have that $\gamma_{Z_{\varepsilon}^{+}}^{-1}\left(y_{f 1}\right)=y_{f 3} \in I^{-}$. The line $\{(x, y) ; x=0\}$ is tangent to the fold points. Therefore we cannot use the Implicit Function Theorem in this case. However, we can obtain a condition for the existence of a sliding/escaping limit cycle studying the intersection of the trajectories of $Z_{\varepsilon}$ in $\Sigma^{-}$with initial conditions at $y_{f 1}, y_{f 2}$ and $y_{f 3}$ with the line $\Lambda=\left\{(x, y) ; x \leq 0, y=y_{f 1}\right\} \subset \Sigma^{-}$, which is a transversal section at the fold point $y_{f 1}$. Considering the smooth vector field $Z_{\varepsilon}^{-}$and the initial conditions $\left(0, y_{f 1}\right),\left(0, y_{f 2}\right)$ and $\left(0, y_{f 3}\right)$ the intersection of the flow of $Z_{\varepsilon}^{-}$with $\Lambda$ define the return maps $S_{0}(\varepsilon), S_{1}(\varepsilon), S_{2}(\varepsilon)$ 
and $S_{3}(\varepsilon)$, respectively, given by

$$
\begin{aligned}
S_{0}(\varepsilon)= & -2 e+\varepsilon\left(2 b_{21}^{-} e-2 v_{2}^{-}\right)+\varepsilon^{2}\left(\frac{1}{2} \pi e\left(c_{11}^{-}+c_{22}^{-}\right)-2\left(v_{1}^{-} b_{22}^{-}-v_{2}^{-} b_{21}^{-}+w_{2}^{-}\right.\right. \\
& \left.\left.+\left(b_{21}^{-}\right)^{2} e-c_{21}^{-} e\right)\right)+\mathcal{O}\left(\varepsilon^{3}\right), \\
S_{1}(\varepsilon)= & -2 e+\varepsilon\left(2 b_{21}^{-} e-2 v_{2}^{-}\right)+\varepsilon^{2}\left(-2\left(v_{1}^{-} b_{22}^{-}-v_{2}^{-} b_{21}^{-}+w_{2}^{-}+\left(b_{21}^{-}\right)^{2} e-c_{21}^{-} e\right)\right. \\
& \left.-\frac{1}{2} \pi e\left(c_{11}^{-}+c_{22}^{-}\right)\right)+\mathcal{O}\left(\varepsilon^{3}\right), \\
S_{2}(\varepsilon)= & -2 e+\varepsilon\left(2 b_{21}^{-} e-2 v_{2}^{-}\right)-\frac{\varepsilon^{2}}{2 b^{2} e}\left(\left(v_{1}^{-}\right)^{2} b^{2}+2 v_{1}^{-} b\left(2 b b_{22}^{-} e+v_{1}^{+}\right)+b^{2} e\left(4 \left(-v_{2}^{-} b_{21}^{-}\right.\right.\right. \\
& \left.\left.\left.+w_{2}^{-}+\left(b_{21}^{-}\right)^{2} e-c_{21}^{-} e\right)-\pi e\left(c_{11}^{-}+c_{22}^{-}\right)\right)+\left(v_{1}^{+}\right)^{2}\right)+\mathcal{O}\left(\varepsilon^{3}\right), \\
S_{3}(\varepsilon)= & -2 e+\varepsilon\left(2 b_{21}^{-} e-2 v_{2}^{-}\right)+\frac{\varepsilon^{2}}{2 b^{2} e}\left(-4 b^{2} e\left(v_{1}^{-} b_{22}^{-}-v_{2}^{-} b_{21}^{-}+w_{2}^{-}\right)-4\left(v_{1}^{-} b+v_{1}^{+}\right)^{2}\right. \\
& \left.+\pi b^{2} e^{2}\left(c_{11}^{-}+c_{22}^{-}\right)+4 b^{2} e^{2}\left(c_{21}^{-}-\left(b_{21}^{-}\right)^{2}\right)\right)+\mathcal{O}\left(\varepsilon^{3}\right) .
\end{aligned}
$$

By hypothesis

$(1-a)$ If $0<c_{11}^{-}+c_{22}^{-}<\left(v_{1}^{-} b+v_{1}^{+}\right)^{2} /\left(2 b^{2} e^{2} \pi\right)$, then $S_{3}<S_{2}<S_{1}<S_{0}$ and system $Z_{\varepsilon}$ admits a sliding cycle of Type I.

$(1-b)$ If $\left.\left(v_{1}^{-} b+v_{1}^{+}\right)^{2}\right) /\left(2 b^{2} e^{2} \pi\right)<c_{11}^{-}+c_{22}^{-}<2\left(v_{1}^{-} b+v_{1}^{+}\right)^{2} /\left(b^{2} e^{2} \pi\right)$, then $S_{3}<S_{1}<S_{2}<$ $S_{0}$, and system $Z_{\varepsilon}$ admits a sliding cycle of Type II.

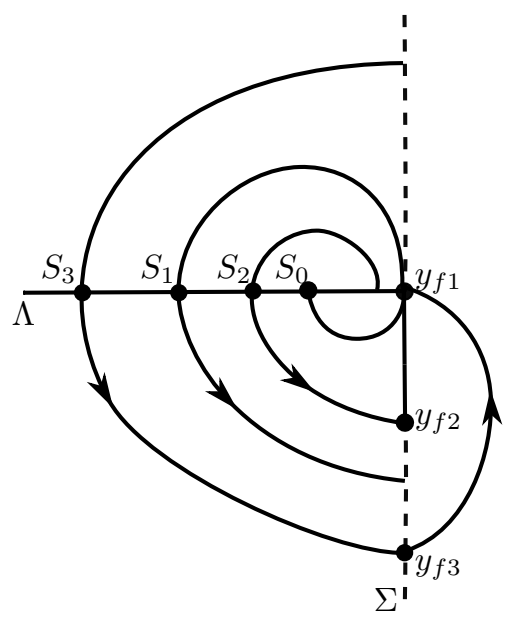

Figure 5. The point $y_{f 3}$ and the return maps $S_{0}(\varepsilon), S_{1}(\varepsilon), S_{2}(\varepsilon)$ and $S_{3}(\varepsilon)$.

Working similarly and assuming that $a<0, d+b e>0$ and $v_{1}^{-} b+v_{1}^{+}>0$ we can conclude that

$(2-a)$ If $\left.-\left(v_{1}^{-} b+v_{1}^{+}\right)^{2}\right) /\left(2 b^{2} e^{2} \pi\right)<c_{11}^{-}+c_{22}^{-}<0$, then system $Z_{\varepsilon}$ admits a escaping cycle of Type I.

$(2-b)$ If $\left.c_{11}^{-}+c_{22}^{-}<-\left(v_{1}^{-} b+v_{1}^{+}\right)^{2}\right) /\left(2 b^{2} e^{2} \pi\right)$, then system $Z_{\varepsilon}$ admits a escaping cycle of Type II.

This completes the proof of Theorem B. 
3.5. Proof of Theorem C. We divide the proof in two steps. In the first we provide an upper bound for the number of crossing limit cycles, and in the second we study the stability of these limit cycles.

Lemma 7. Assuming that $b_{11}^{-}=-b_{22}^{-}$the function $M_{1}$, given in (9), has at most one simple zero. Moreover, there exists choice of parameters for which this upper bound is reached.

Proof. Consider $M_{1}\left(y_{0}\right)$ given in (9) and assume that $b_{11}^{-}=-b_{22}^{-}$, then we obtain

$$
M_{1}\left(y_{0}\right)=2 v_{1}^{-}+\frac{2 v_{1}^{+}}{b}+\frac{d\left(b_{11}^{+}+b_{22}^{+}\right)}{\xi^{2}}-\frac{\left(b_{11}^{+}+b_{22}^{+}\right)\left(d^{2}+\xi^{2} y_{0}^{2}\right) \arccos \left(\frac{2 d^{2}}{d^{2}+\xi^{2} y_{0}^{2}}-1\right)}{2 y_{0} \xi^{3}}
$$

Proceeding with the change of coordinates $y_{0}=d s_{0} / \xi$ and writing $k_{0}=2 v_{1}^{-}+2 v_{1}^{+} / b+$ $d\left(b_{11}^{+}+b_{22}^{+}\right) / \xi^{2}$ and $k_{1}=-d\left(b_{11}^{+}+b_{22}^{+}\right) /\left(2 \xi^{2}\right)$ we get

$$
\frac{d}{\xi} s_{0} M_{1}\left(s_{0}\right)=k_{0} s_{0}+k_{1}\left(s_{0}^{2}+1\right) \arccos \left(1-\frac{2}{s_{0}^{2}+1}\right) .
$$

So, denoting

$$
\begin{aligned}
& f_{0}\left(s_{0}\right)=s_{0} \\
& f_{1}\left(s_{0}\right)=\left(s_{0}^{2}+1\right) \arccos \left(1-\frac{2}{s_{0}^{2}+1}\right),
\end{aligned}
$$

and computing their Wronskians we obtain

$$
\begin{aligned}
& W\left(f_{0}\right)\left(s_{0}\right) \quad=1 \\
& W\left(f_{0}, f_{1}\right)\left(s_{0}\right)=-2 s_{0}+\left(s_{0}^{2}-1\right) \arccos \left(1-\frac{2}{s_{0}^{2}+1}\right) .
\end{aligned}
$$

Let $\widetilde{W}_{1}\left(s_{0}\right)=W_{1} /\left(s_{0}^{2}-1\right)$. Therefore $\frac{d \widetilde{W}_{1}}{d s_{0}}\left(s_{0}\right)=8 s_{0}^{2} /\left(\left(s_{0}^{2}-1\right)^{2}\left(s_{0}^{2}+1\right)\right)$ which is strictly positive for all $s_{0}>0$. So, $\widetilde{W}_{1}$ is strictly increasing and $W_{1}$ has at most one zero. The existence of a sliding limit cycle follows from Theorem B. In Example 4.2 we present a piecewise linear vector field that exhibits a crossing/sliding limit cycle.

In the previous case the stability of the crossing limit cycle is given by the following result.

Lemma 8. The crossing limit cycle of (2) is unstable (resp. stable) if $v_{1}^{-}+\frac{v_{1}^{+}}{b}>0$ (resp. $\left.v_{1}^{-}+\frac{v_{1}^{+}}{b}<0\right)$.

Proof. The stability of the crossing limit cycle is given by the sign of $M_{1}$. Indeed, $M_{1}\left(y_{0}\right)=$ $M_{1}^{-}\left(y_{0}\right)-M_{1}^{+}\left(y_{0}\right)$, let $y_{0}^{*} \in \mathbb{R}^{+}$such that $M_{1}\left(y_{0}\right)=0, M_{1}\left(y_{0}\right)>0$ for $y_{0}<y_{0}^{*}$, and $M_{1}\left(y_{0}\right)<0$ for $y_{0}>y_{0}^{*}$. Therefore, the crossing limit cycle is unstable. If $M_{1}\left(y_{0}\right)<0$ for $y_{0}<y_{0}^{*}$ and $M_{1}\left(y_{0}\right)>0$ for $y_{0}>y_{0}^{*}$, then the crossing limit cycle is stble. By (12) we have that

So, the lemma follows.

$$
\lim _{y_{0} \rightarrow 0^{+}} M_{1}\left(y_{0}\right)=2\left(v_{1}^{-}+\frac{v_{1}^{+}}{b}\right) .
$$




\section{Final Remarks AND some examples}

In this section we provide two examples of piecewise linear vector fields, the first one admitting three crossing limit cycles and the second one with a sliding and a crossing limit cycle.

4.1. Example 1. Consider the following piecewise linear vector field $Z=\left(Z^{+}, Z^{-}\right)$where

$$
\begin{aligned}
Z^{-}(x, y)= & \left(\begin{array}{cc}
0 & -1 \\
1 & 0
\end{array}\right)\left(\begin{array}{l}
x \\
y
\end{array}\right)+\left(\begin{array}{c}
0 \\
0.55
\end{array}\right) \\
& +\varepsilon\left(\left(\begin{array}{cc}
-1 & 0 \\
0 & -1
\end{array}\right)\left(\begin{array}{l}
x \\
y
\end{array}\right)+\left(\begin{array}{c}
-2.65 \\
0
\end{array}\right)\right)
\end{aligned}
$$

and

$$
Z^{+}(x, y)=\left(\begin{array}{cc}
1 & -1 \\
1.01 & -1
\end{array}\right)\left(\begin{array}{l}
x \\
y
\end{array}\right)+\left(\begin{array}{c}
0 \\
0.1
\end{array}\right)+\varepsilon\left(\left(\begin{array}{cc}
0.21 & 0 \\
0 & 0
\end{array}\right)\left(\begin{array}{l}
x \\
y
\end{array}\right)\right) .
$$

The first order Melnikov function associated to this system is given by

$$
\begin{aligned}
M_{1}\left(y_{0}\right)= & \frac{-1}{400 y_{0}}\left(-242 \pi-800 \pi y_{0}^{2}+420\left(y_{0}^{2}+1\right) \arccos \left(\frac{2}{y_{0}^{2}+1}-1\right)\right. \\
& \left.+\left(400 y_{0}^{2}+121\right) \arccos \left(\frac{242}{400 y_{0}^{2}+121}-1\right)+840 y_{0}\right),
\end{aligned}
$$

which has three zeros $y_{0}=1, y_{0}=2$ and by the Newton-Kantorovich method, see [2], we have the third zero in the neighborhood of $y_{0}=3.82781$, see Figure 6 . Each zero corresponds to a crossing limit cycle with alternating stability. In this case, the highest amplitude crossing limit cycle is unstable and the infinity is stable.

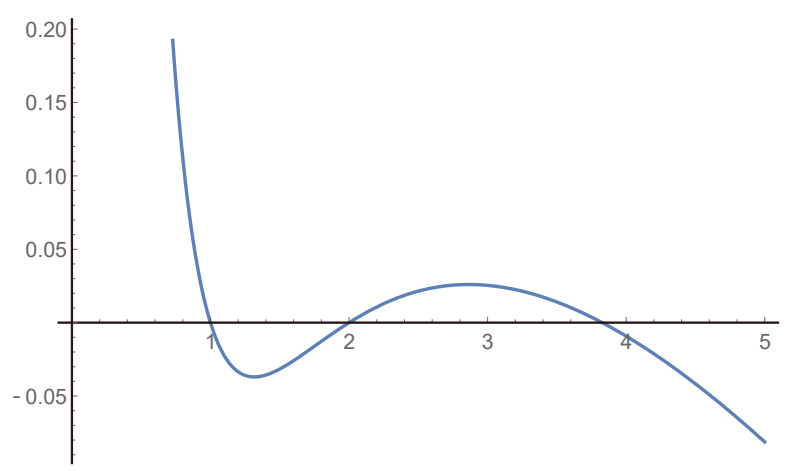

Figure 6. Graphic of $M_{1}$. Each zero correspond to a crossing limit cycle. 
4.2. Example 2. Consider the following piecewise linear vector field $Z=\left(Z^{+}, Z^{-}\right)$where

$$
\begin{aligned}
Z^{-}(x, y)= & \left(\begin{array}{cc}
0 & -1 \\
1 & 0
\end{array}\right)\left(\begin{array}{l}
x \\
y
\end{array}\right)+\left(\begin{array}{l}
0 \\
1
\end{array}\right)+\varepsilon\left(\left(\begin{array}{cc}
1 & 0 \\
0 & -1
\end{array}\right)\left(\begin{array}{l}
x \\
y
\end{array}\right)+\left(\begin{array}{c}
0.2 \\
0
\end{array}\right)\right) \\
& +\varepsilon^{2}\left(\left(\begin{array}{cc}
0.03 & 0 \\
0 & 0.02
\end{array}\right)\left(\begin{array}{l}
x \\
y
\end{array}\right)\right) \\
Z^{+}(x, y)= & \left(\begin{array}{cc}
-1 & -1 \\
2 & 1
\end{array}\right)\left(\begin{array}{l}
x \\
y
\end{array}\right)+\left(\begin{array}{l}
0 \\
2
\end{array}\right)+\varepsilon\left(\left(\begin{array}{cc}
1.5 & 0 \\
0 & -0.4
\end{array}\right)\left(\begin{array}{l}
x \\
y
\end{array}\right)+\left(\begin{array}{c}
-0.5 \\
0
\end{array}\right)\right)
\end{aligned}
$$

The associated first order Melnikov function is given by

$$
M_{1}\left(y_{0}\right)=2.2-\left(0.1 y_{0}+\frac{1.6}{y_{0}}\right) \arccos \left(\frac{8}{0.25 y_{0}^{2}+4}-1\right) .
$$

The graph of $M_{1}$ is given by Figure 7 , the crossing limit cycle is located in a neighborhood of $y_{0}=7.94622$, is repealer and the infinite is attractor.

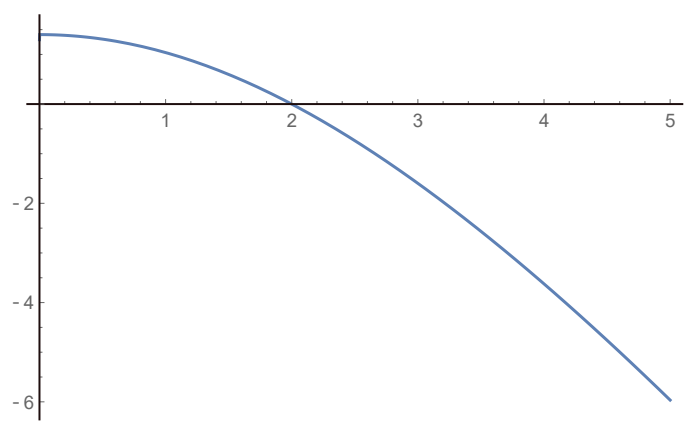

Figure 7. Graphic of $M_{1}$. The unique zero of $M_{1}$ correspond to a repealer crossing limit cycle.

\section{ACKNowledgements}

JL is partially supported by the Ministerio de Economía, Industria y Competitividad, Agencia Estatal de Investigación grant MTM2016-77278-P (FEDER), the Agència de Gestió d'Ajuts Universitaris i de Recerca grant 2017 SGR 1617, and the European project DynamicsH2020-MSCA-RISE-2017-777911. JLC is partially supported by Goiás Research Foundation (FAPEG) and CAPES. DDN is partially supported by a FAPESP grant 2016/11471-2. DJT is partially supported by PROCAD/CAPES grant 88881.0 68462/2014-01 and by CNPqBrazil. 


\section{REFERENCES}

[1] A. Andronov, A. Vitt And S. Khaikin, Theory of Oscillations Pergamon Press, Oxford, 1966.

[2] I.K. Argyros, Convergence and applications of Newton-type iterations., Springer Science and Business Media, 2008.

[3] M. di Bernardo, C.J. Budd, A. R. Champneys and P. Kowalczyk, Piecewise-Smooth Dynamical Systems: Theory and Applications, Appl. Math. Sci. Series 163, Springer-Verlag, London, 2008.

[4] C. Buzzi, C. Pessoa And J. Torregrosa, Piecewise linear perturbations of a linear center, Discrete Continuous Dyn. Syst. 9 (2013), 3915-3936.

[5] C. Christopher and C. Li, Limit cycles of differential equations, Birkhauser Verlag, Basel, 2007.

[6] E. Freire, E. Ponce, F. Rodrigo And F. Torres, Bifurcation sets of continuous piecewise linear systems with two zones, Int. J. Bifurcation and Chaos 8 (1998), 2073-2097.

[7] F. Giannakopoulos And K. Pliete, Planar systems of piecewise linear differential equations with a line of discontinuity, Nonlinearity 14 (2001), 1611-1632.

[8] M.R.A. Gouveia, J. Llibre, And D.D. Novaes, On limit cycles bifurcating from the infinity in discontinuous piecewise linear differential system, Appl. Math. Comput. 271 (2015), 365-374.

[9] M. HAN AND W. Zhang, On Hopf bifurcation in non-smooth planar systems, J. of Differential Equations 248 (2010), 2399-2416.

[10] S.M. HUAN AND X.S. YANG, On the number of limit cycles in general planar piecewise linear systems, Disc. Cont. Dynam. Sys. 32 (2012), 2147-2164.

[11] S.J. Karlin And W.J. Studden, T-Systems: With Applications in Analysis and Statistics, Pure Appl. Math., Interscience Publishers, New York, London, Sidney, 1966.

[12] J. Llibre, M. OrdóÑez AND E. Ponce, On the existence and uniqueness of limit cycles in planar continuous piecewise linear systems without symmetry, Nonlinear Analysis: Real World Applications 19 (2012), 325-335.

[13] J. Llibre, D. D. Noves, And M. A. TeixeIrA, Limit cycles bifurcating from the periodic orbits of a discontinuous piecewise linear differentiable center with two zones, Int. J. Bifurcation and Chaos 25 (2015), 1550144 (11 pages).

[14] J. Llibre, D. D. Noves, And M. A. Teixeira, Maximum number of limit cycles for certain piecewise linear dynamical systems, Nonlinear Dynamics 82 (2015), 1159-1175.

[15] J. Llibre AND E. Ponce, Hopf bifurcation from infinity for planar control system, Publicacions Matemàtiques 41 (1997), 181-198.

[16] J. Llibre AND E. Ponce, Three nested limit cycles in discontinuous piecewise linear differential systems with two zones, Dynamics of Continuous, Discrete and Impulsive Systems, Serie B 19 (2012), 325-335

[17] D. D. NovaEs, Number of limit cycles for some non-generic classes of piecewise linear differential systems, In Extended Abstracts Spring 2016: Nonsmooth Dynamics. Trends in Mathematics 8, Birkhäuser/Springer, Cham, 2017, 135-139.

[18] M. A. TeIXEIRA, Stability conditions for discontinuous vector fields, Journal of Differential Equations 88 (1990), 15-29.

[19] M. A. TeIXeIrA, Generic singularities of discontinuous vector fields, An. Acad. Brasil. Ciênc. 53 (1981), 257-260.

[20] Filippov, A. F., Differential equations with discontinuous righthand sides, Kluwer Academic Publishers Group, Dordrecht, 18, 1988.

[21] Novaes, D. D. And Torregrosa, J. A., On extended Chebyshev systems with positive accuracy, J. Math. Anal. Appl. 448 (2016), 171-186.

1 Institute of Mathematics and Statistics of Federal University of Goís, Avenida Esperança s/n, Campus Samambaia, 74690-900, Goin̂nia, Goiás, Brazil

Email address: joao.lopes@ifg.edu.br

Email address: djtonon@ufg.br

2 Departament de Matematiques, Universitat Autònoma de Barcelona, 08193 Bellaterra, Barcelona, Catalonia, Spain

Email address: jllibre@mat.uab.cat 
3 Departamento de Matematica, Universidade Estadual de Campinas, Rua Sérgio Buarque de Holanda, 651, Cidade Universitária, 13083-859, Campinas, São Paulo, Brazil

Email address: ddnovaes@ime.unicamp.br 